\title{
Development of Heat Flow Measurement using Thermal Probe Method in Divertor Simulator MAP-II
}

\author{
Kiminori KURIHARA, Shinichiro KADO ${ }^{1)}$, Hiroto MATSUURA ${ }^{2)}$, Kyu-Sun $\mathrm{CHUNG}^{3)}$, Taiichi \\ SHIKAMA, Yohei IIDA, Filippo SCOTTI, Yosuke KUWAHARA and Satoru TANAKA \\ School of Engineering, The University of Tokyo, Bunkyo, Tokyo 113-8656, Japan \\ ${ }^{1)}$ High Temperature Plasma Center, The University of Tokyo, Kashiwa, Chiba 277-8568, Japan \\ ${ }^{2)}$ Graduate School of Engineering, Osaka Prefecture University, Sakai, Osaka 599-8531, Japan \\ ${ }^{3)}$ electric Probe Applications Laboratory (ePAL), Hanyang University, Seoul 133-791, Korea
}

(Received 4 December 2006 / Accepted 5 April 2007)

\begin{abstract}
The method of measuring heat flow $Q$ as a function of the sheath potential $V$ is investigated in order to improve the time response of the thermal probe method. We found that the time constant $\tau$ for reaching a steady state in thermal phenomena was $123 \pm 18 \mathrm{~s}$. The result of $\tau$ indicated that the applicable method for measuring the $Q-V$ characteristic was biasing the thermal probe in a stepwise manner, and this was verified experimentally. Although there existed a $V$-dependent offset of the $Q-V$ curve deduced from the temperature gradients against that calculated from the ion current, a procedure to deduce the ion temperature $T_{\mathrm{i}}$ is developed. The experimental error in $T_{\mathrm{i}}$ was too large, and was caused by the error in $Q$. Thus we have to improve the accuracy of the $Q$ measurement.
\end{abstract}

(C) 2007 The Japan Society of Plasma Science and Nuclear Fusion Research

Keywords: Thermal probe, $Q-V$ characteristic, Time constant, Ion temperature, Divertor simulator MAP-II

DOI: $10.1585 /$ pfr.2.S1082

\section{Introduction}

The thermal probe method is a recently proposed plasma diagnostic. In this method the heat flow into a probe tip $(Q)$ is measured as a function of the sheath potential $(V)$. Based on this $Q-V$ characteristic, one can principally deduce various plasma parameters such as the negative ion density [1] and the ion temperature $T_{\mathrm{i}}[2,3]$. We adopt the method of $Q$ measurement using the temperature gradient as described in [4] in order to apply the thermal probe to the fusion edge/divertor plasmas, where $Q$ is considerably larger than that of laboratory plasmas. The time resolution of the thermal probe is poor in nature because of the long transient time of thermal phenomena [5]. Therefore, in this paper we apply a method that involves improved time response.

\section{Principles}

\subsection{Heat transfer}

It can safely be assumed in the present condition that the thermal conduction governs the heat flow in a thermal probe. The temperature profile can then be described by the 1-dimensional heat conduction equation. This equation can be solved under the two boundary conditions: (i) Stationary heat flow, (ii) the temperature at the heat sink doesn't change. Then the leading term of the solution at one point $\left(x=x_{1}\right)$ is given by Eq. (1),

author'se-mail: kimi@flanker.q.t.u-tokyo.ac.jp kado@q.t.u-tokyo.ac.jp

$$
\begin{aligned}
T_{\mathrm{p}}\left(x_{1}, t\right)= & T_{\mathrm{p}}\left(x_{1}, \infty\right) \\
& +\left\{T_{\mathrm{p}}\left(x_{1}, 0\right)-T_{\mathrm{p}}\left(x_{1}, \infty\right)\right\} \exp (-t / \tau),
\end{aligned}
$$

where $T_{\mathrm{p}}$ is the temperature in the thermal probe, $\tau=$ $4 L^{2} / \alpha \pi^{2}$ the time constant for reaching the steady state, $L$ the length of the thermal probe, $\alpha$ the thermal diffusivity of the probe tip material. $\tau$ depends only on the length for a given material. We use $\alpha=5.37 \times 10^{-5} \mathrm{~m}^{2} / \mathrm{s}$ for a molybdenum probe.

\section{$2.2 Q-V$ characteristic}

Based on the plasma sheath theory, $Q(V)[\mathrm{W}]$ from the positive and negative charges in the plasma is described by [6] as Eq. (2),

$$
\begin{aligned}
Q(V)= & I_{\text {is }}(V) \times\left\{\left(2 T_{\mathrm{i}}-V\right)\left(1-R_{\mathrm{E}}\right)+E_{\mathrm{rec}}\right\} \\
& +\left\{I_{\mathrm{p}}(V)-I_{\text {is }}(V)\right\} \times 2 T_{e},
\end{aligned}
$$

where $T_{\mathrm{i}}$ and $T_{\mathrm{e}}$ are the ion and electron temperature in $\mathrm{eV}$, respectively, $R_{\mathrm{E}}$ is the ion energy reflection coefficient, $I_{\mathrm{p}}(V)$ the probe current, and $E_{\text {rec }}$ the surface recombination energy in $\mathrm{eV}$. Although we use the values of $R_{\mathrm{E}}$ interpolating from those in the ALLADIN database [7] in Eq. (2), $R_{\mathrm{E}}$ can also be deduced from the $Q-V$ characteristic [8]. The ion saturation current $I_{\mathrm{is}}(V)$ is deduced by fitting the $I_{\mathrm{p}}(V)$ data points in the ion dominant region to the linear function while the electron current is evaluated as $\left\{I_{\mathrm{p}}(V)-\right.$ $\left.I_{\text {is }}(V)\right\} . Q(V)$ can also be deduced from the temperature gradient along the probe tip under the steady state, $\Delta T_{\mathrm{p}}(V$, 


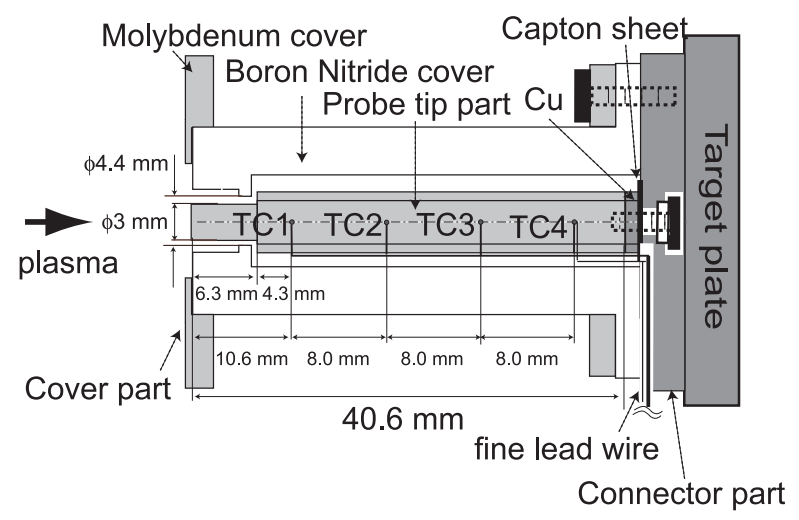

Fig. 1 Schematic view of the thermal probe. The thermal probe consists of a probe tip, a cover, and a connector.

$\infty) / \Delta x$, using Eq. (3) as,

$$
Q(V)=-\kappa \frac{\Delta T_{\mathrm{p}}(V, \infty)}{\Delta x} S,
$$

where $\kappa$ is the thermal conductivity of the probe tip material and $S$ its cross section. In the case of molybdenum, $\kappa$ is $138 \mathrm{Wm} / \mathrm{K}$, and $S$ of our thermal probe is $1.9 \times 10^{-5} \mathrm{~m}^{2}$. There are two methods for the $Q-V$ characteristic measurement. One is to change $V$ in a stepwise manner (stepwise method) and the other is to sweep $V$ continuously (sweeping method). While the stepwise method obtains only a finite number of data points of the $Q-V$ curve, $\Delta T_{\mathrm{p}}(V, \infty)$ can be deduced from the temperature evolution at the transient state by fitting it to Eq. (1). On the other hand, although the sweeping method can obtain a continuous $Q-V$ curve, it is required to measure $\Delta T_{\mathrm{p}}(V, \infty)$ directly.

\section{Apparatus}

The experiments were conducted using low-pressure helium dc-arc discharges in the linear steady-state divertor simulator MAP-II at the University of Tokyo. The typical parameters of MAP-II helium plasma are $T_{\mathrm{i}}=0.4$ $0.7 \mathrm{eV}$ measured by He II spectrometry, and $n_{\mathrm{e}}=10^{17}$ $2 \times 10^{18} \mathrm{~m}^{-3}$ and $T_{\mathrm{e}}=3-15 \mathrm{eV}$ measured using a Langmuir probe [9]. At the downstream chamber in MAP-II, the thermal probe is mounted on the target plate, which is cooled by a chiller unit, and two external coolant tubes are inserted in the drain path, which enables us to maintain the temperature of the inlet of the heat sink at $20^{\circ} \mathrm{C}$.

The thermal probe consists of three parts: a probe tip part, a cover part, and a connector part as shown in Fig. 1. The probe tip is made of cylindrical molybdenum, whose length is $40.6 \mathrm{~mm}$ and diameters are $3 \mathrm{~mm}$ (left part) and $5 \mathrm{~mm}$ (right part). The probe tip is equipped with four thermo-couples for the $\Delta T_{\mathrm{p}}$ measurement, and with a fine leads wire for biasing as well as the $I_{\mathrm{p}}$ measurement. The thermal probe can obtain not only the $Q-V$ but also the $I$ $V$ characteristics simultaneously. The thermal probe and its cables are shielded from the plasma by a boron nitride. The

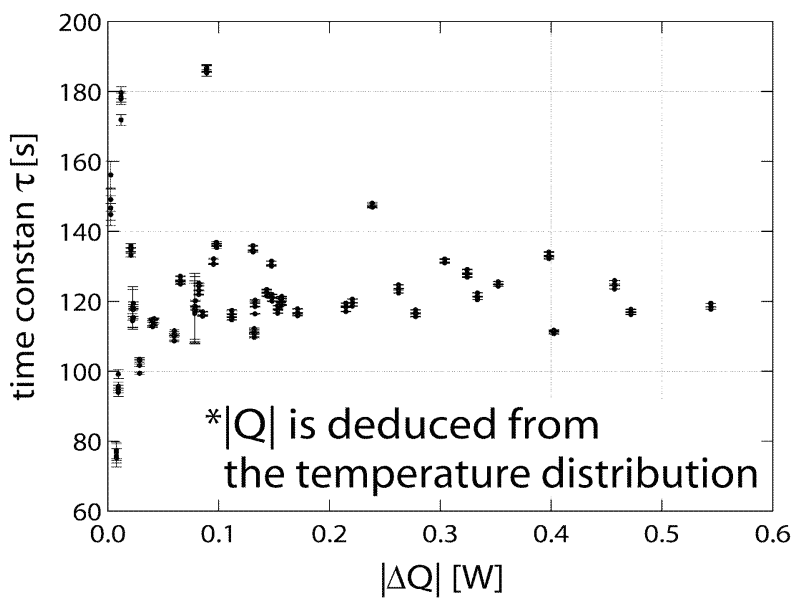

Fig. 2 Time constant $\tau$ is independent of $|\Delta Q|$ and the scatter of $\tau$ is small for $|\Delta Q|>0.1 \mathrm{~W}$.

boron nitride insulator is further covered with a molybdenum cap. The double capton sheets provide electrical insulation between the probe tip part and the connector part in order to bias the thermal probe tip only.

\section{Results and Discussion}

\subsection{Time constant $\tau$}

One can see from Eq. (1) that $\tau$ is independent of $|\Delta Q|$. This fact is supported experimentally as can be seen in Fig. 2 where the scatter of $\tau$ became small for $|\Delta Q|>$ $0.1 \mathrm{~W}$. This result suggests that the change in $V$ should be higher than a certain threshold. The value of $\tau$ obtained from Fig. 2 was $123 \pm 18 \mathrm{~s}$, which was longer than $12 \mathrm{~s}$ calculated from $\tau=7.55 \times 10^{-3} L^{2}[\mathrm{~s}]$, with $L=40.6 \mathrm{~mm}$. This significant difference comes from the fact that the practical probe length should be interpreted as that of an equivalent probe made of uniform material including the thermal contact resistance of the whole system, as shown in Fig. 3. The practical length of this virtual probe $L_{\mathrm{vir}}$ was $128 \pm 14 \mathrm{~mm}$ calculated from $L=1.15 \times 10 \tau^{0.5}[\mathrm{~mm}]$.

$L_{\mathrm{vir}}$ can also be deduced from the $T_{\mathrm{p}}$ distribution in the thermal probe. We think of the heat sink as the cross points of several $T_{\mathrm{p}}$ distributions as shown in Fig. 3. This figure also shows that the $T_{\mathrm{p}}$ distribution was not linear due to the deviation of TC3 or TC4. But since this deviation was observed to be systematic, the model in which the heat conduction was dominant in the probe could be applied by determining which set of thermo-couples was appropriate to the true $T_{\mathrm{p}}$ distribution. $L_{\mathrm{vir}}$ from the set of TC1-TC2TC3, $136 \mathrm{~mm}$, was more consistent with that from $\tau$ than that of TC1-TC2-TC4, $221 \mathrm{~mm}$. We suspected that the $L_{\mathrm{vir}}$ of the former set is more consistent with that determined from $\tau$. Therefore, we tentatively adopted this set throughout this paper. However, further investigation would be needed about the adequacy of the idea of the virtual probe. 


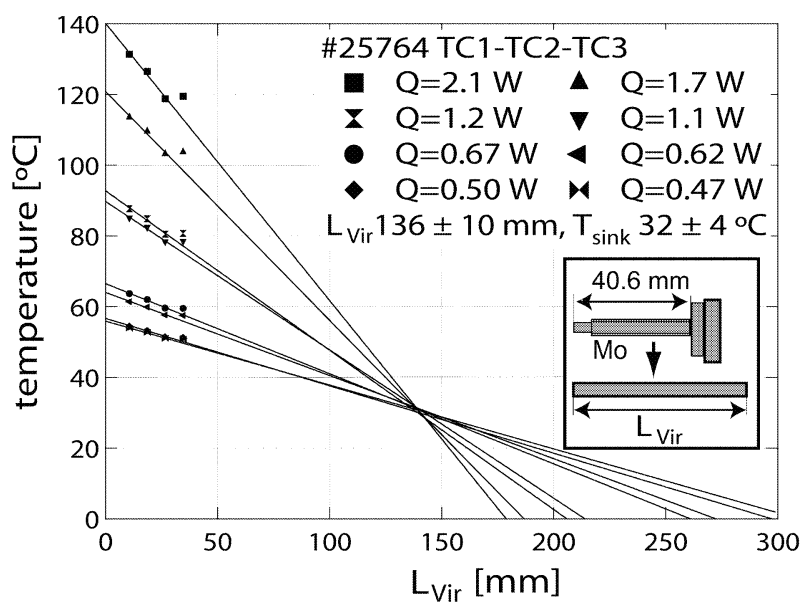

Fig. $3 L_{\text {vir }}$ is determined as the cross points of several temperature distributions corresponding to various heat flow obtained by TC1-TC2-TC3.

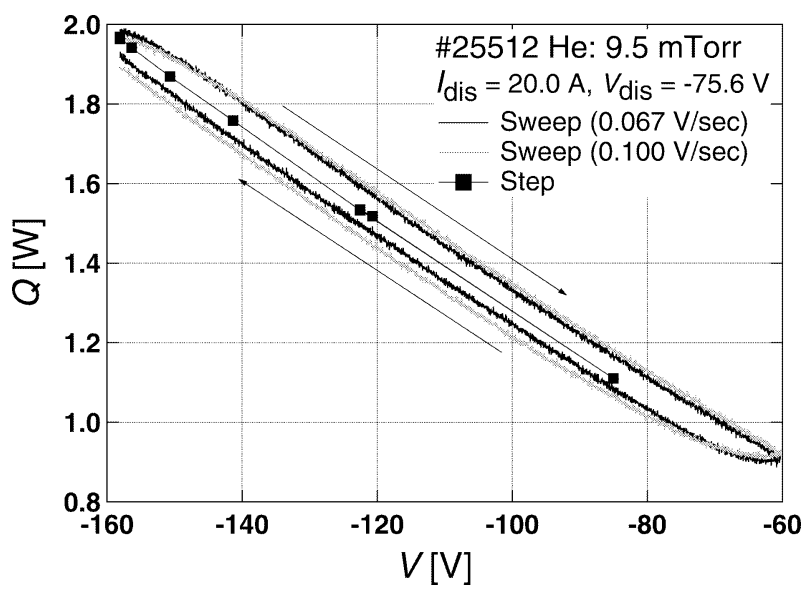

Fig. 4 Comparison of $Q-V$ characteristic obtained by the sweeping method and stepwise method.

\section{2 $Q-V$ characteristic}

The result of the $Q-V$ characteristic obtained by the two methods is shown in Fig. 4. In the sweeping method, $V$ was swept at two speeds: $0.067 \mathrm{~V} / \mathrm{s}$ and $0.100 \mathrm{~V} / \mathrm{s}$. Since the values between at $\mathrm{d} V / \mathrm{d} t>0$ and at $\mathrm{d} V / \mathrm{d} t<0$ had hysteresis, the measurement was not performed under the steady state. We can conclude that the stepwise method is applicable under our present status against the long $\tau$ of our thermal probe. But there is the possibility that the sweeping method can work for a narrow $V$ region in the case of small $Q$ plasma as shown in [3]. Since $|\Delta Q|$ per $V$ is small in the small $Q$ plasma, $\Delta T_{\mathrm{p}}(V, \infty)$ is little different from the transient state. In addition to that, hysteresis occurs in the narrow $V$ measurement less than in the wide $V$ measurement.

The $Q-V$ characteristic shown in Fig. 5 was measured under the following discharge conditions: helium 9.5 mTorr, discharge current $I_{d i s}=30.0 \mathrm{~A}, V_{d i s}=-80.9 \mathrm{~V}$, while $n_{\mathrm{e}}$ and $T_{\mathrm{e}}$ measured by the thermal probe itself are

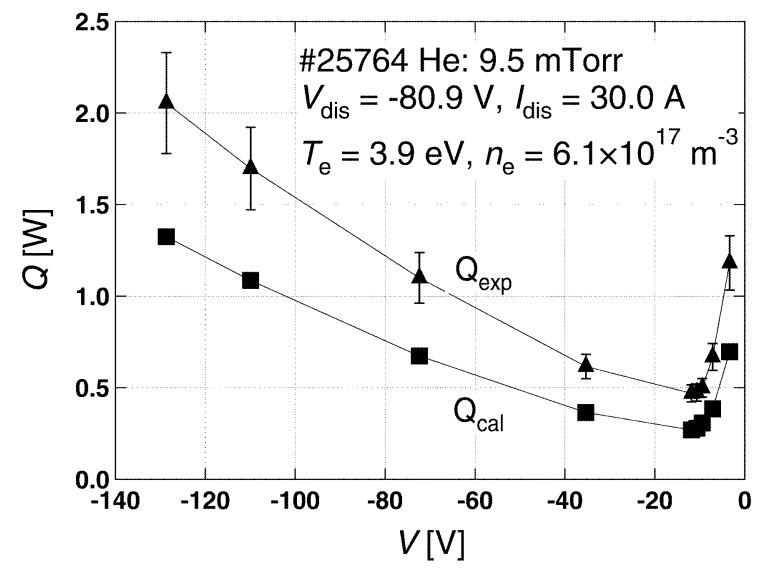

Fig. $5 Q-V$ characteristic measured by the stepwise method at He $9.5 \mathrm{mTorr}$, the discharge current $I_{\mathrm{dis}}=30.0 \mathrm{~A}, V_{\mathrm{dis}}=$ $-80.9 \mathrm{~V}, n_{\mathrm{e}}$ and $T_{\mathrm{e}}$ are $6.1 \times 10^{-7} \mathrm{~m}^{-3}$ and $3.9 \mathrm{eV}$.

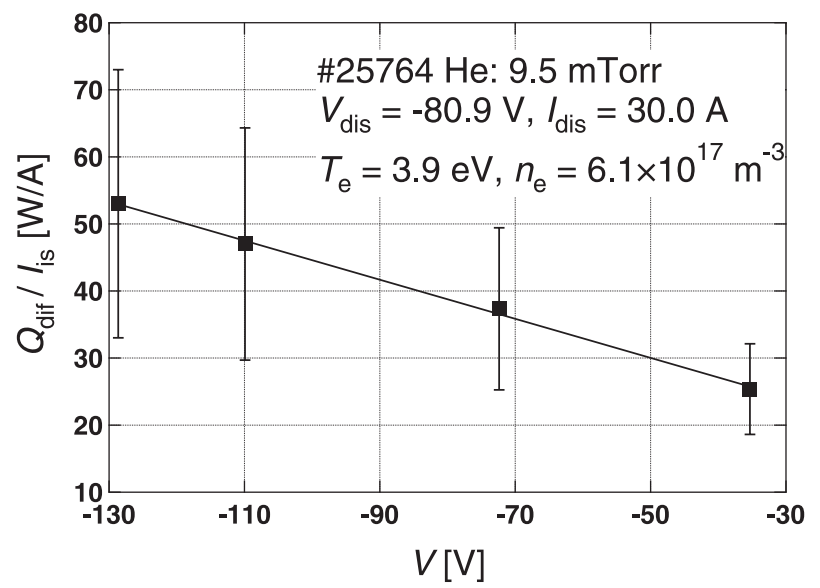

Fig. $6 Q_{\text {dif }} / I_{\text {is }}$ in the ion dominant region shows linearity to $V$. This dependence determines $Q_{\text {dif }}$, which is used in the analysis for $T_{\mathrm{i}}$ and effective $R_{\mathrm{E}}$.

$6.1 \times 10^{17} \mathrm{~m}^{-3}$ and $3.9 \mathrm{eV}$, respectively. $Q_{\text {exp }}$ was deduced from $\Delta T_{\mathrm{p}} / \Delta x$ of TC1-TC2-TC3. $Q_{\text {cal }}$ was calculated from Eq. (2), assuming $T_{\mathrm{i}}=0$ and using the experimental data for $I_{\mathrm{p}}(V)$. The assumption of $T_{\mathrm{i}}$ is based on the typical value of MAP-II. The value of $E_{\text {rec }}$ is $20.4 \mathrm{eV}$ considering the $24.6 \mathrm{eV}$ ionization energy of helium and the $4.2 \mathrm{eV}$ work function of molybdenum. Comparing $Q_{\exp }$ with $Q_{\text {cal }}$, there existed an obvious difference, $Q_{\text {dif }}$, having a dependence on $V$. Although the physical process of $Q_{\text {dif }}$ is now under investigation, $T_{\mathrm{i}}$ and $R_{\mathrm{E}}$ can be deduced assuming the linear $V$ dependence of $Q_{\text {dif }} / I_{\text {is }}$ in the ion dominant region as shown in Fig. 6. Adding the contribution of $Q_{\text {dif }}$ to the Eq. (2) in the ion dominant region we have

$$
Q(V)=I_{\mathrm{is}}(V) \times\left\{\left(2 T_{\mathrm{i}}-V\right)\left(1-c R_{\mathrm{E}}\right)+E_{\mathrm{rec}}+a V+b\right\},
$$

where $a$ and $b$ are the fitting coefficients determined from the $V$ dependence of $Q_{\text {dif }} / I_{\text {is }}, c$ is the calibration factor for 
$R_{\mathrm{E}}$ in a practical sense, introduced to make use of the $V$ dependence of $R_{\mathrm{E}}$ in the database. The analysis consists of the following 4 procedures: (i) $a$ and $b$ are obtained assuming the linear $V$ dependence of $Q_{\text {dif }} / I_{\text {is }}$, (ii) assuming $T_{\mathrm{i}}=0$, the $Q_{\exp }-V$ curve is fitted to Eq. (4) to obtain $c$, (iii) using $c$ obtained in (ii), the $Q_{\text {exp }}-V$ curve is fitted to Eq. (4) to obtain $T_{\mathrm{i}}$, and (iv) the iteration for fitting $Q_{\exp }-V$ curve is conducted until $c$ and $T_{\mathrm{i}}$ converge. The results of $T_{\mathrm{i}}$ and $c$ where the fitting region are $V=-129$ to $-35 \mathrm{~V}$ were $0.17 \pm 41.6 \mathrm{eV}$ and $1.0 \pm 0.8$. The error in $T_{\mathrm{i}}$ consists of the fitting error in $Q_{\exp }$ and $T_{\mathrm{i}}$ and the experimental errors in $a, b$, and $c$, whose contributions to the error in $T_{\mathrm{i}}$ are $13,0.2,13,19$, and $31 \mathrm{eV}$, respectively. The reason why the experimental error in $c$ is the biggest of all factors is that it is propagated from those not only in $Q_{\exp }$ (ii), but also in $a$ and $b$ (i). The error in $T_{\mathrm{i}}$ is determined mainly by the propagation from the error in $Q_{\exp }$, because the error in $Q_{\text {exp }}$ influences those in $a, b$, and $c$. In order to improve the accuracy of $T_{\mathrm{i}}$, we have to decrease the error in $Q_{\exp }$. To reduce the error in $T_{\mathrm{i}}$ as much as to the typical value of MAP-II, in other word, the error in $T_{\mathrm{i}}$ is $100 \%$, we decrease the error in $Q_{\exp }$ about one-fiftieth from the present value. Therefore, reliable in-situ calibration of $Q_{\exp }$ [10] is required, which is a subject for future study. It is then necessary to increase the measurement points in order to reduce the effect of the fitting error of $a$ and $b$.

\section{Conclusion}

The method of measuring the $Q-V$ characteristic has been improved. We found that $\tau$ was $123 \pm 18 \mathrm{~s}$ of our thermal probe, this result indicating that the stepwise method is more applicable than the sweeping method. Comparing $L_{\mathrm{vir}}$ from the $T_{\mathrm{p}}$ distribution with that from $\tau$, the $T_{\mathrm{p}}$ distribution deduced from the set of TC1-TC2-TC3 was adopted. $Q_{\text {exp }}$ deduced from the result of TC1-TC2-TC3 had $V$-dependent offset $Q_{\text {dif }}$ compared to $Q_{\text {cal }}$. Although the physical process of $Q_{\text {dif }}$ is under investigation, we could deduce $T_{\mathrm{i}}$ and effective $R_{\mathrm{E}}$ assuming $Q_{\mathrm{dif}} / I_{\mathrm{is}}$ is linearly dependent on $V$. Their errors in the current system are still large due to the measurement error in $Q_{\text {exp }}$, which suggests the requirement of in-situ calibration. In the present development stage, the method is being verified in low $T_{\mathrm{i}}$ steady-state laboratory plasma. If the $T_{\mathrm{i}}$ is comparably higher than the recombination energy, $E_{\mathrm{rec}}$, the method's greater reliability will have been demonstrated.

\section{Acknowledgments}

This work was supported in part by a NIFS Collaborative Research Program (NIFS04KOAB009) directed by the second author.

[1] E. Stamate, H. Sugai and K. Ohe, Appl. Phys. Lett. 80, 3066 (2002).

[2] H. Matsuura and K. Michimoto, Contrib. Plasma Phys. 44, 677 (2004).

[3] K. Kurihara, S. Kado, H. Matsuura et al., Trans. Fusion Sci. Tech. 51, 250 (2007).

[4] H. Matsuura, T. Jida and S. Kado, in Proc. 27th Int. Conf. on Reactive Plasmas (Veldhoven, 2005) topic number 8.

[5] P.C. Stangeby, Physics of plasma-Wall Interactions in Controlled Fusion, eds. D.E. Post and R. Behrisch (Plenum Press, New York, 1984) p. 41.

[6] IAEA AMDIS ALADDIN database: http://www-amdis. iaea.org/ALADDIN/

[7] S. Masuzaki, N. Ohno and S. Takamura, J. Nucl. Mater. 223, 286 (1995).

[8] S. Kado Y. Iida, S. Kajita et al., J. Plasma Fusion Res. 81, 810 (2005). http://www.jspf.or.jp/Journal/2005.html

[9] M. Osakabe, Y. Takeiri, T. Takanashi et al., Rev. Sci. Instrum. 72, 586 (2001).

[10] B. Koch, W. Bohmeyer, G. Fussmann et al., J. Nucl. Mater. 290-293, 653 (2001). 